\title{
Resonance Effect of Nanofibrous Membrane for Sound Absorption Applications
}

\author{
Klara Kalinova \\ Additional information is available at the end of the chapter
}

http://dx.doi.org/10.5772/intechopen.70361

\begin{abstract}
Nanofibrous layers have unique acoustic properties due to the large specific surface area of the nanofibers, where viscous losses may occur and also the ability of the nanofiber layer to resonate at its own frequency. The resonance membrane is then, upon impact of sound waves of low frequency, brought into forced vibrations, whereby the kinetic energy of the membrane is converted into thermal energy by friction of individual nanofibers, by the friction of the membrane with ambient air, and possibly with other layers of material arranged in its proximity, and part of the energy is also transmitted to the frame, by which means the vibrations of the resonance membrane are damped. When sound waves hit the nanofiber membrane, they introduce forced vibrations in the case of resonance which have maximal amplitude. The principle of the technology is achieved by the synergy of perforated plate in the form of a cavity resonator with nanofibrous layer in the form of resonant membrane. The parameters of the resonant nanofibrous membrane together with the shape and volume of the perforations then determine which sound frequencies will be damped and to what extent.
\end{abstract}

Keywords: membrane, nanofibers, sound absorption, foil

\section{Introduction}

The confusion between sound insulation and sound absorption is often phenomenon. Soundabsorbing materials play an indispensable part in controlling noise generated within a room or in reverberant areas. Although such materials are highly effective as sound absorbers, they are relatively poor sound insulators because of their soft, porous, and lightweight construction. Sound insulation prevents sound traveling from one place to another such as between apartments in a building. A part of sound energy is absorbed, the next part is reflected, and the rest is transmitted to the second room. The sound attenuation is due to the air viscosity, nonreversible deformation of material, and the thermal conduction between the fibers and the 
air. The sound absorption also depends on structure characteristics, that is, fiber arrangement, fiber fineness, density of fiber, porosity, and so on. The sound absorption of lower frequencies becomes the main problem of this acoustic section.

Nanofibrous layers have unique acoustic properties due to the large specific surface area of the nanofibers, where viscous losses may occur and also the ability of the nanofiber layer to resonate at its own frequency. The resonance membrane is then, upon impact of sound waves of low frequency, brought into forced vibrations, whereby the kinetic energy of the membrane is converted into thermal energy by friction of individual nanofibers, by the friction of the membrane with ambient air, and possibly with other layers of material arranged in its proximity, and part of the energy is also transmitted to the frame, by which means the vibrations of the resonance membrane are damped. When sound waves hit the nanofiber membrane, they introduce forced vibrations in the case of resonance which have maximal amplitude.

\subsection{Membrane resonators}

Materials based on resonance principle can be divided into three groups: arrangements behaving as vibrating membranes, arrangements behaving as vibrating plates, and arrangements consisting in the principle of Helmholtz resonators.

The work [1] uses a mechanic analogy of an acoustic resonance system consisting of an acoustic mass $m_{a}$ connected to an acoustic plasticity $c_{a r}$ the movement of which is dampened by an acoustic resistance $R_{a}$. The behavior of the membrane (plate) can be compared to the behavior of a corpus with a certain mass flexibly connected to a spring (represented by an air cushion, of by the air in material pores). Assuming that the elements representing the mass are perfectly stiff and the elements representing the flexibility have no mass, this problem can be compared to the theory of linear circuits in the field of electrical engineering, where the coils are considered as having no capacity, condensers having no inductivity, and resistors being purely ohmic [2]. As in the field of electrical engineering, where the notion of electrical impedance is introduced, which is defined as the ratio between the voltage and current, a similar variable can be introduced for acoustic systems - the acoustic impedance $Z$. It is defined as the ratio of the pressure affecting the system and the volumetric rate at which the system vibrates thanks to the effect of the abovementioned force. For individual elements, apply the following:

$$
Z_{m}=j \omega m_{a}, Z_{R}=R_{a}, Z_{c}=\frac{1}{j \omega c_{a}}
$$

where $j$ is an imaginary unit, $\omega$ the angular frequency, in $\mathrm{s}^{-1}$.

Assuming that the system is not damped $\left(R_{a}=0\right)$, it meets the equation

$$
j \omega m_{a}+\frac{1}{j \omega c_{a}}=0,
$$

the resonance of the system according to Ref. [1] then occurs at the frequency 


$$
f_{r}=\frac{1}{2 \pi} \sqrt{\frac{1}{m_{a} c_{a}}}=\frac{1}{2 \pi} \sqrt{\frac{\rho}{m_{s q} d}},
$$

where $c$ is the speed of sound propagation through the environment, in $\mathrm{m} \mathrm{s}^{-1}, \rho$ is the air density, in $\mathrm{kg} \mathrm{m}^{-3}, m_{s q}$ is the surface density of the membrane, in $\mathrm{kg} \mathrm{m}^{-2}$, and $d$ is the thickness of the air cushion in $\mathrm{m}$.

The influence of the surface density of the membrane on the resonance frequency of the system, or the sound absorption coefficient that increases with this characteristics, is also apparent. We can also notice the shift in the maximums of the sound absorption coefficient toward lower frequencies.

A thin circular membrane is defined in Ref. [3] as a structure that arises by stretching, for example, a thin homogeneous elastic film with a constant surface density to a rigid circular frame. The membrane gains its rigidity by means of this stretching induced by radially acting force. The resonance frequency of a thin circular membrane is defined using the relation

$$
f_{i}=\frac{a_{0, i}}{2 \pi R} \sqrt{\frac{v}{m_{s q}}}
$$

where $a_{0, i}$ are constants of symmetric vibrations of the circular membrane for $i$-modes, $R$ is the membrane diameter, in $\mathrm{m}$, and $v$ radially acting stretching force related to the unit of the frame diameter (membrane tension), in $\mathrm{N} \mathrm{m}^{-1}$.

From the above, it shows that the resonance frequency of the membrane decreases with its increasing surface density. Membrane resonators based on a resonant principle of a nanofibrous layer function effectively as slim lightweight absorbing solutions. Contrary to conventionally used microscale sound absorbers, sound-absorbing membranes based on submicron fibers show a higher absorption abilities-due to the possibility of resonating on its own resonant frequency, the nanofibrous membrane is able to absorb critical lower sound frequencies. These unique properties come from the nature of nanofibrous layers, that is, small fibrous diameter (respectively, high specific surface area) and high porosity. This makes it possible to reach higher viscous loss inside the material and consequently to dissipate the acoustic energy. Nanofibrous elements and optimal rigidity of the membrane itself then allow an acoustic system to vibrate more efficiently $[4,5]$. Resonant nanofibrous membranes of insignificant thickness are prepared from different polymer solutions in the form of electrospun nanofibers captured on a substrate layer via electrospinning method.

The theoretical bases of sound absorption characteristics that the paper deals with are studies performed by Sakagami et al. The study [6] focuses on a membrane-type sound absorber. To analyze the absorption mechanism, the solution is rearranged in a form which points out the contribution from each element of the membrane. The effects of the parameters of the sound absorption system are discussed in the light of the calculated results. Also, the method used for predicting the peak frequency and the peak value of the oblique-incident absorption coefficient 
of the membrane-type sound absorber is presented and satisfactorily explains the relationship between the absorption characteristics and the parameters.

Resonant behavior of a microperforated panel for various perforation ratios in comparison with a panel-/membrane-type absorber is presented in Ref. [7], considering back-wall surface effect. The effectiveness of a fiber-based sound absorbance material involves several parameters such as porosity, tortuosity, fiber diameter, surface density, and thickness [8]. The optimal material types and structural characteristics of such membranes are in the deep interest of researchers, and although some have been proposed, it still remains as a subject of research. Kalinová has demonstrated that the resonance frequency of polyvinyl alcohol (PVA) nanofibrous acoustic membranes decreases with an increasing surface density and the average diameter of the nanofibers [5]. Rabbi et al. sandwiched polyacrylonitrile (PAN) and polyurethane (PUR) nanofibrous membrane between two nonwoven layers of polyester (PET) and wool. All materials with electrospun membrane(s) were found to significantly increase its absorbance. Moreover, the effect of nanofiber layer's number and its surface density was investigated [9]. Asmatulu et al. tested the sound absorbance property of electrospun polyvinyl chloride (PVC) mat of different thickness and with fiber diameters ranging from a few hundred nanometers to a few microns. When the fiber diameter goes beyond $500 \mathrm{~nm}$, the sound absorbance shift toward the lower frequency with a thicker mesh but absorption coefficients remain the same [4].

\subsection{Helmholtz's resonators}

Helmholtz's resonators are acoustic systems that consist of a swinging air plug and a connected air volume. It can have a variety of forms: an empty wine bottle, corpus of a string instrument, bass reflex enclosures of loudspeakers, and wall coverings made of perforated panel. These acoustic systems can be arranged either separately or jointly to the perforated board, which is mounted to a certain distance from the wall [10].

In the study [11], the variable system of sound absorption power by the chairs in the lowfrequency range was examined. As the results of scale model experiments (1/10 scale) in the reverberation room, the absorption power was controlled in the low-frequency range by the opening and closing of holes of the resonator. The diameter of holes, a neck's length, and a cavity volume of the seat were evaluated. The result was obtained for 125 or $250 \mathrm{~Hz}$ by changing the cavity volume of the seat in the experiment.

An acoustical structure consisting of a large-scale isolated resonator with a large-diameter cylindrical cavity has been studied in the work [12]. This resonator differs from the classical Helmholtz's resonator where the cavity is only several millimeters in diameter and lined with a sound-absorbing material. The impedance of the cavity and the impedance of the volume of the resonator are calculated. Calculations show that the sound energy is absorbed by resonators made of sound-reflecting materials. Absorption is of a resonant character with the resonant frequency at $60 \mathrm{~Hz}$. A resonator measuring $200 \times 200 \mathrm{~cm}$, with the cavity diameter of $50 \mathrm{~cm}$ and the distance to the rigid surface being $30 \mathrm{~cm}$, absorbs $3.5 \mathrm{~m}^{2}$ of sound energy at the resonant frequency. At very low frequencies, changes in the imaginary parts of both cavity and radiation impedances occur along with the increase in the cavity diameter and frequency. 
The study [13] deals with the effect of orifice geometry on the resonance frequency of Helmholtz's resonators. Helmholtz's theoretical formula for calculating resonant frequency $f_{\mathrm{H}}$ is as follows:

$$
f_{\mathrm{H}}=\frac{c}{2 \pi} \sqrt{\frac{a}{V}}
$$

where $c$ is the sound velocity, $a$ is the diameter of orifice, and $V$ is the volume of cavity. Further, Sondhauss's calculation of resonant frequency $f_{S}$ with the correction $\delta=4 a / 3 \pi$ is then

$$
f_{H}=\frac{c}{2 \pi} \sqrt{\frac{A}{V(l+2 \delta)}},
$$

where $A$ is the orifice area and $l$ is the orifice thickness.

\subsection{Measuring methods}

Vibration phenomena can be investigated by the noninvasive optical methods. One of the most widely used methods for vibration measurement and analysis is laser vibrometry that can be combined with the high-speed camera. This approach can be seen in different application fields, for example, the development and monitoring of high-speed milling devices [14-21]. Nabavi describes the utilization of the particle image velocimetry technique to measure the velocity of the standing waves within an air-filled rigid-walled square channel subjected to acoustic standing waves. The data were compared with the analytical results obtained from the time-harmonic solution of the wave equation [22].

The resonant effect of nanofibrous membrane has been studied by means of high-speed digital camera in the author's paper [23]. The study attempted to predict the sound absorption behavior of the PVA nanofibrous membrane in comparison with the homogeneous membrane structure using an experimental setup involving a high-speed camera. The membrane has been exposed to plane sinusoidal sound wave and its deflection was picked by the high-speed digital camera. The resonant peaks of oscillating nanofibrous membrane as well as homogeneous membrane occur (see Figure 1). The recent study [24] shows how except for the lowest frequencies (first resonance peak), the resonant behavior of the membrane is affected by the resonance of the tube when the effect of mass per unit area on resonance frequencies of the membrane placed in an open and closed tubes is investigated.

Two-microphone impedance measurement tube type 4206 is used to measure the absorption coefficient in the frequency ranges from $100 \mathrm{~Hz}$ to $6.4 \mathrm{kHz}$. This is achieved by measuring the incident and reflected components of random noise, which is generated inside the tube. From the incident and reflected components of the sound pressure at two microphone positions, the frequency response functions are calculated due to the cross-spectrum of the two microphone signals. Using these values, the sound absorption coefficient can be determined. An apparatus is used to determine the sound absorption coefficient of laboratory circular samples with a 


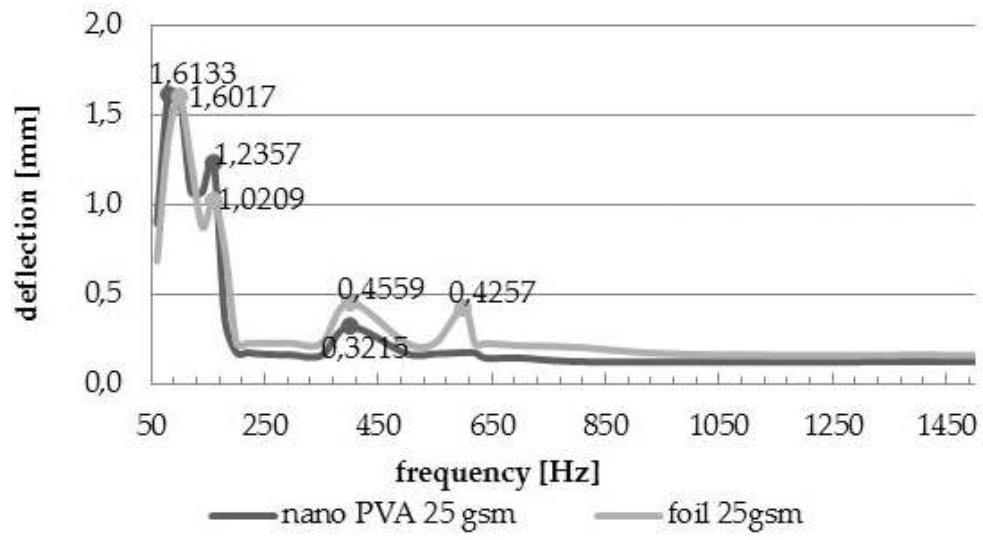

Figure 1. The resonance peaks of nanofibers compared with foil (took over author's paper 23).

diameter of $100 \mathrm{~mm}$ for a frequency range of $100-1600 \mathrm{~Hz}$ and $29 \mathrm{~mm}$ for a frequency range of 500-6400 Hz, according to the standard ASTM E1050-08.

Due to the fact that the quad perforations in the plate were almost the same size as the diameter of the small tube of the measuring apparatus, the samples were measured only in a large tube with a diameter of $100 \mathrm{~mm}$ for a limited frequency range of 100-1600 Hz. These frequencies, however, cover the area particularly focusing on middle and lower frequencies.

\section{Acoustic element design}

\subsection{Production of nanofibrous layer for membrane resonator design}

For the production of nanofibrous membranes, roller electrospinning method (nanospider machine) was used. In this method, there is a roller that is connected to a high voltage supplier, and at the top of the roller there is a counterelectrode that was grounded. Taylor cones are created on the roller surface toward counterelectrode (Figure 2). Individual nanofibrous layer of very low basis weight of about $0.1-2 \mathrm{~g} / \mathrm{m}^{2}$ is not self-supporting. That is why the nanofibers are deposited on a thin supporting textile. This carrier has to be sound permeable with a low basis weight of about $20-50 \mathrm{~g} / \mathrm{m}^{2}$. Process parameters such as roller speed, distance between the electrodes, voltage, and so on are set for an optimal nanofiber diameter and the basis weight of nanofibrous membrane.

For the production of PA6 nanofibrous membranes, the cord electrospinning method was used [25]. In this method, the cord was connected to a high voltage supply, and at the top of the cord there was a counterelectrode, which was grounded. The liquid polymeric material is applied onto the cord around its whole circumference, and then the application means moving reversibly along the active spinning zone of the cord and the process of electrostatic spinning of the liquid polymeric material is started. Taylor cones were created on the cord surface toward the counterelectrode. 


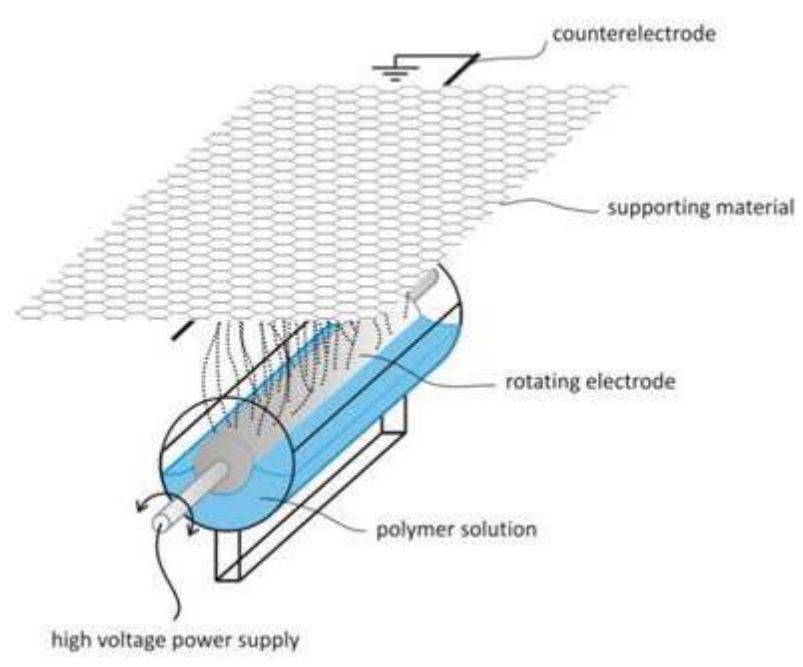

Figure 2. Schematic diagram of roller electrospinning method used for PVA nanofibrous membrane production.

\subsection{Cavity resonator together with nanofibrous resonant membrane}

The principle of the technology is achieved by the synergy of the perforated plate in the form of a cavity resonator with nanofibrous layer in the form of a resonant membrane. The resonant nanofibrous membrane is arranged on the surface of the cavity resonator, to which it is fixedly attached, for example, glued or laminated, and so on. Its parts, which overlap the orifices leading into the cavities of the cavity resonator, constitute separate resonant surfaces, whereby the resonant frequency of each of them is determined, apart from the overall properties of the resonant membrane, also by their size and shape. Upon impact of sound waves, these resonant surfaces are brought into forced vibrations, which are subsequently damped by friction in the inner structure of the resonant membrane, by the friction of the resonant membrane against ambient air, and possibly against other layers of the material arranged in its proximity, wherein part of the kinetic energy of the resonating membrane is transmitted to the cavity resonator. Moreover, friction in the inner structure of the resonant membrane is further increased by the fact that the neighboring resonant surfaces can vibrate with mutually different periods or deviation.

At the same time, it is possible-while maintaining the thickness of the acoustic element-to damp sound frequencies which could be normally damped by the cavity resonator with extremely large air gap. In order to obtain the required sound-absorbing properties, the resonant membrane can be arranged on both opposing surfaces of the cavity resonator.

The acoustic element is based on a quad hollow plate (see Figure 3) whose reverse side is covered by a thin carrier layer with a nanofibrous membrane which to a certain extent protects the frame against mechanical damage. For the final application in the room acoustic, the space between the nanofibrous membrane covering the thin perforated plate and the wall or ceiling 

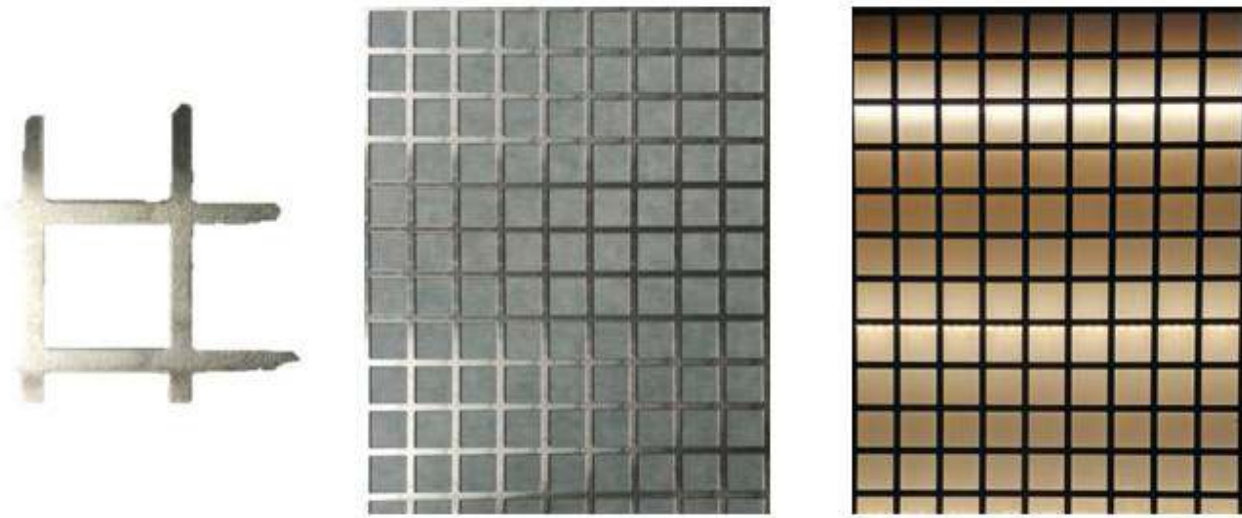

Figure 3. Components used to design the acoustic elements - aluminum quad hollow plate $9 / 11$ (size of perforation is $9 \times$ $9 \mathrm{~mm}$, span of perforation is $11 \times 11 \mathrm{~mm}$ ) with a thickness of $1 \mathrm{~mm}$ (left) covered with a nanofiber layer (middle) lighted (right).

(20-50-mm air gap in the mentioned experimental) is of huge benefit to the new technology. It can be used for the installation of lighting, audio speakers or heating, and so on. The soundabsorbing means can be used, for example, for the production of acoustic bodies, interior blinds, tiling, ceilings, screens, and separating walls for interiors, or, as the case may be, segment or profile elements for the transportation industry (paneling of cabin).

Figure 3 (on the right) shows the final lighted prototype of acoustic system based on nanofibrous membrane covering the thin perforated plate. The resonance frequency of the acoustic system is then determined especially by dimensions of plate perforations, by the size and shape of the inlet orifices, and by its material and thickness of the plate.

Table 1 shows the calculation of resonant frequency for each of quad perforated plates that have been studied at the experimental section of this work.

Firstly, due to the fact that the quad perforations in the plate were almost the same size as the diameter of the small tube of the measuring apparatus, the samples were measured only in a

\begin{tabular}{lll}
\hline Quad hollow plate (quad size in $\mathrm{mm} /$ quad span in $\mathrm{mm})$ & $f_{\mathrm{H}}(\mathrm{Hz})$ & $f_{\mathbf{S}}(\mathrm{Hz})$ \\
\hline $3 / 5$ & 2682 & 2467 \\
$4 / 6$ & 2581 & 2462 \\
$5 / 7$ & 2473 & 2415 \\
$8 / 10$ & 2190 & 2219 \\
$9 / 11$ & 2112 & 2155 \\
$10 / 12$ & 2040 & 2095 \\
$25 / 30$ & 1290 & 1369 \\
\hline
\end{tabular}

Table 1. Calculated resonant frequency of separate perforated plates based on Helmoltz's $\left(f_{\mathrm{H}}\right)$ formula (5) and Sondhauss's $\left(f_{\mathrm{S}}\right)$ formula $(6)$. 
large tube with a diameter of $100 \mathrm{~mm}$ for a limited frequency range of 100-1600 Hz. These frequencies, however, do not cover the resonant frequencies of separate perforated plates calculated in Table 1. Secondly, in the case of nanofibrous layer in a form of resonant membrane, the measurement of membrane tension $v$ for resonant frequency calculation according to formula 4 is impossible because of the low tension together with non-homogeneous nanofibrous layer. It is why the resonant frequency of nanofibrous membrane has been determined by the optical method [24] where the first resonant peak was detected around $100 \mathrm{~Hz}$. Then, the results of nanofibers-covering perforated plate and the separate perforated plate are compared only by way of sound absorption curves.

\section{Sound absorption results}

In this section, the sound absorption measurements of acoustic means with nanofibrous membrane are shown. Two-microphone impedance measurement tube type 4206 was used to measure the sound absorption coefficient in a limited frequency range of 100-1600 Hz.

The following figures show a graphs of sound absorption coefficients $\alpha$ in dependence on the frequency of sound for separate aluminum plate having different size of orifices and spacing between quad orifices, which is deposited in different distances from the wall (i.e., separate Helmholtz resonator), as well as for sound-absorbing means comprising this perforated plate, whose surface is overlapped by the resonant membrane formed by the layer of nanofibers from polyamide 6 (PA6) having a basis weight of $0.2 \mathrm{~g} \mathrm{~m}^{-2}$ deposited on a thin carrier having a basis weight of $25 \mathrm{~g} \mathrm{~m}^{-2}$. One of the configurations is filled by a foam or a fleece having a thickness of $20 \mathrm{~mm}$.

The individual perforated plate and the same perforated plate covered by a thin carrier with nanofibers have been compared and are shown in Figure 4. The huge growth of sound absorption of middle frequencies can be seen. Starting with $500 \mathrm{~Hz}$, the sound absorption curve of nanofibers improved element is constant contrary of the unstable curve of individual
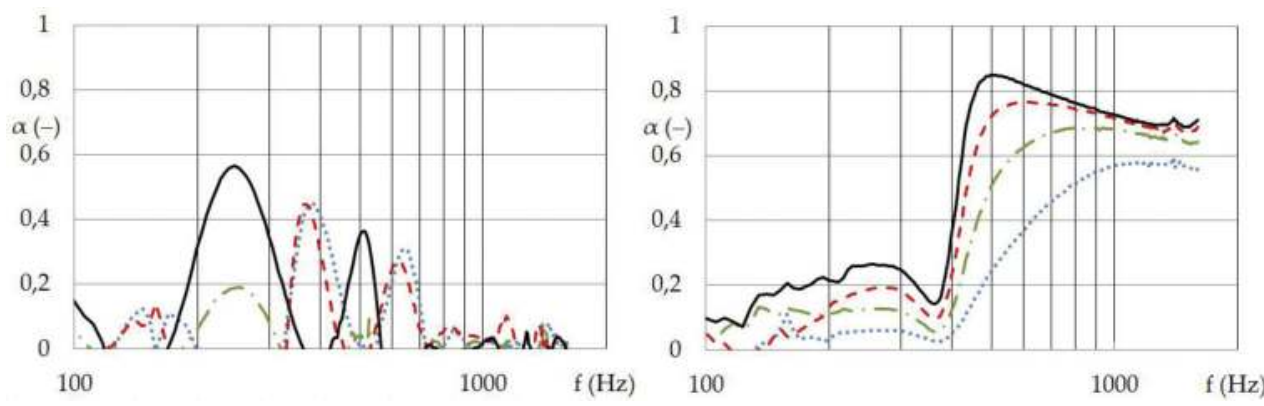

Figure 4. Frequency dependence of the sound absorption coefficient; quad hollow plate 9/11 (side of quad perforation is $9 \mathrm{~mm}$, span of quad perforation is $11 \mathrm{~mm}$ ) with a thickness of $1 \mathrm{~mm}$ with an air gap of 20 (blue - dotted), 30 (green - dashdotted), 40 (red - dashed), and $50 \mathrm{~mm}$ (black) on the left. Nanofibrous membrane of $0.2 \mathrm{~g} / \mathrm{m}^{2}$ on a carrier of $25 \mathrm{~g} / \mathrm{m}^{2}$ covering the same perforated plate (quad 9/11) on the right. 
perforated plate. For the verification of nanofibrous membrane efficient, the individual nanofiber carrier without nanofibers has been evaluated and is shown in Figure 5. Then, it is evident that the carrier-covering perforated plate improves the sound absorption of high frequencies but it does not provide wide-frequency efficiency as well as nanofibers improving plate.

Quad perforated plate of different sizes and spans has been evaluated and is shown in Figure 6. When the size of the perforation is $9 \times 9 \mathrm{~mm}$ and the span of the perforation is $11 \times 11 \mathrm{~mm}$, then it is marked $(9 / 11)$.

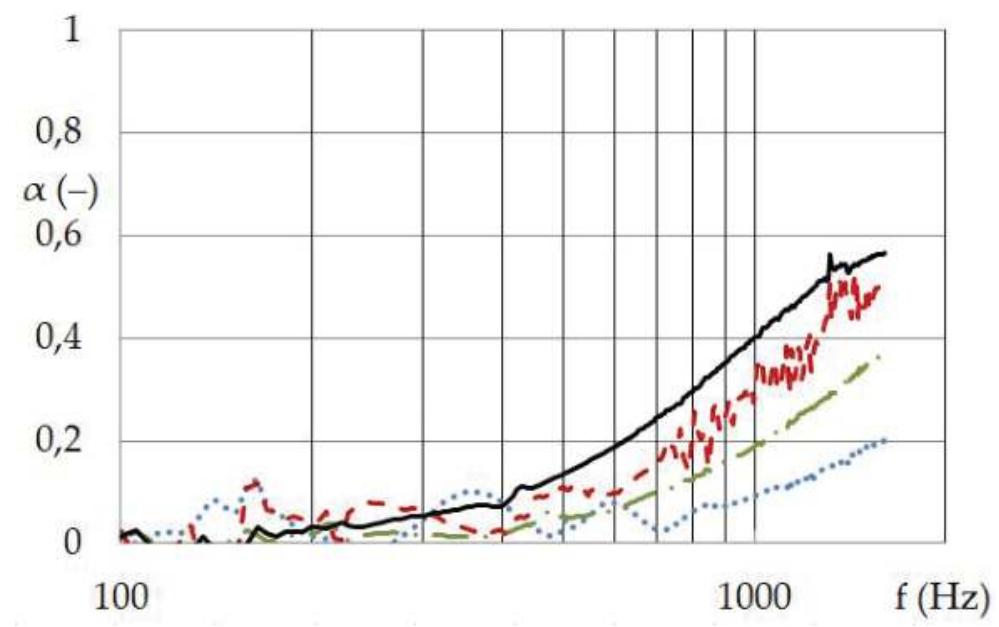

Figure 5. Frequency dependence of the sound absorption coefficient; individual carrier of $25 \mathrm{~g} / \mathrm{m}^{2}$ covering the perforated plate (quad 9/11) with an air gap of 20 (blue - dotted), 30 (green - dash-dotted), 40 (red - dashed), and 50 mm (black) between the acoustic element and the wall.
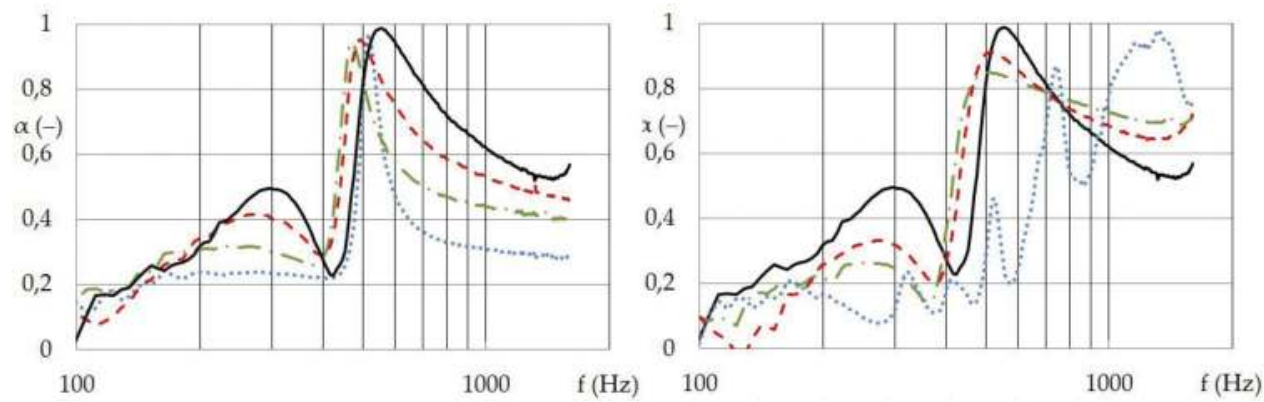

Figure 6. Frequency dependence of the sound absorption coefficient; nanofibrous membrane of $0.2 \mathrm{~g} / \mathrm{m}^{2}$ on a carrier of $25 \mathrm{~g} / \mathrm{m}^{2}$ covering the quad perforated plate of different size with a thickness of $1 \mathrm{~mm}$ with an air gap of $50 \mathrm{~mm}$. Quad perforated plate of 3/5 (blue - dotted), 4/6 (green - dash-dotted), 5/7 (red - dashed), and 8/10 (black) of side/span (left). Quad perforated plate of 8/10 (black), 9/11 (green - dash-dotted), 10/12 (red - dashed), and 25/30 (blue - dotted) of side/ span (right). 
With an increasing size of quad hole, the sound absorption achieves the wide-frequency efficiency generally as can be seen in Figure 6. The best arrangement of quad hole seems to be 9-mm side of quad and the span of $11 \mathrm{~mm}$ (9/11), where the nanofibrous resonant membrane interacts with the perforated panel to achieve optimal parameters of the acoustic system.

Due to two effects, the large specific surface area of the nanofibers and also the ability of the nanofibrous layer to resonate at its own frequency, the nanofibrous membrane achieves broadband sound absorption compared to the narrowband effect of homogeneous foil on the same perforated plate (see Figure 7). Starting with $500 \mathrm{~Hz}$, the sound absorption curve of nanofibers improved element is constant contrary of the unstable curve of foil improved perforated plate.

When the perforated plate is improved by the nanofibrous membrane on each of both sides, then the sound absorption of higher frequencies falls slightly (see Figure 8). Then, the
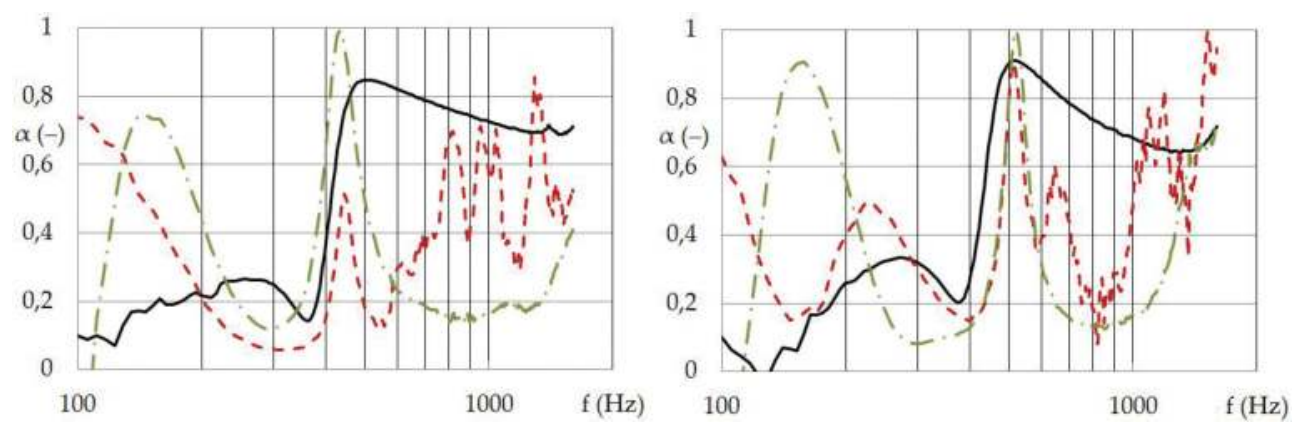

Figure 7. Frequency dependence of the sound absorption coefficient; quad perforated plate of 9/11 (left) and 10/12 (right) is covered by the nanofibrous membrane of $0.2 \mathrm{~g} / \mathrm{m}^{2}$ on a carrier of $25 \mathrm{~g} / \mathrm{m}^{2}$ (black) or foil of $7 \mathrm{~g} / \mathrm{m}^{2}$ (red - dashed) or foil of $40 \mathrm{~g} / \mathrm{m}^{2}$ (green - dash-dotted). The air gap between the 1- $\mathrm{mm}$ thick panel and the wall is $50 \mathrm{~mm}$.
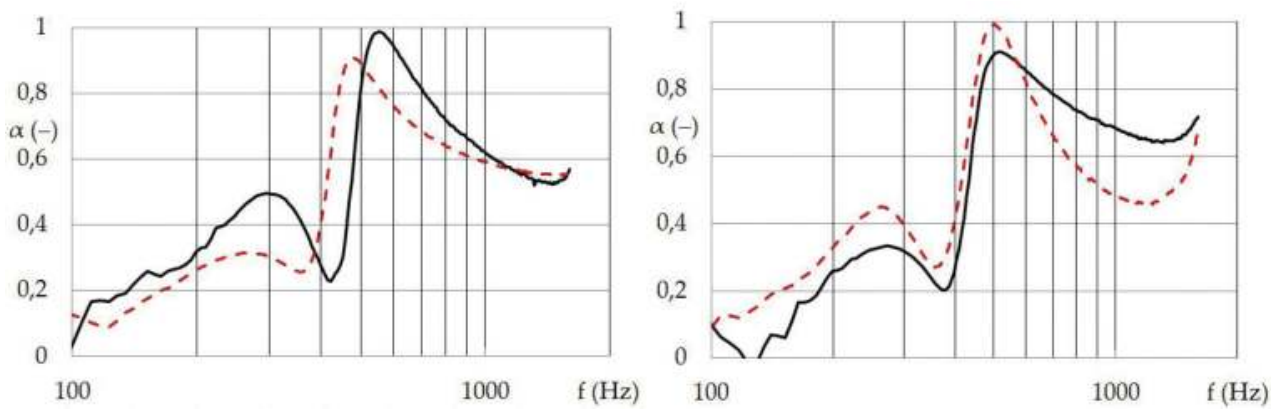

Figure 8. Frequency dependence of the sound absorption coefficient; quad perforated plate of 8/10 (left) and 10/12 (right) is covered at the top by the single nanofibrous membrane of $0.2 \mathrm{~g} / \mathrm{m}^{2}$ on a carrier of $25 \mathrm{~g} / \mathrm{m}^{2}$ (black) or it is covered by the nanofibrous membrane of $0.2 \mathrm{~g} / \mathrm{m}^{2}$ on a carrier of $25 \mathrm{~g} / \mathrm{m}^{2}$ (red - dashed) from both sides. The air gap between the 1-mm thick panel and the wall is $50 \mathrm{~mm}$. 

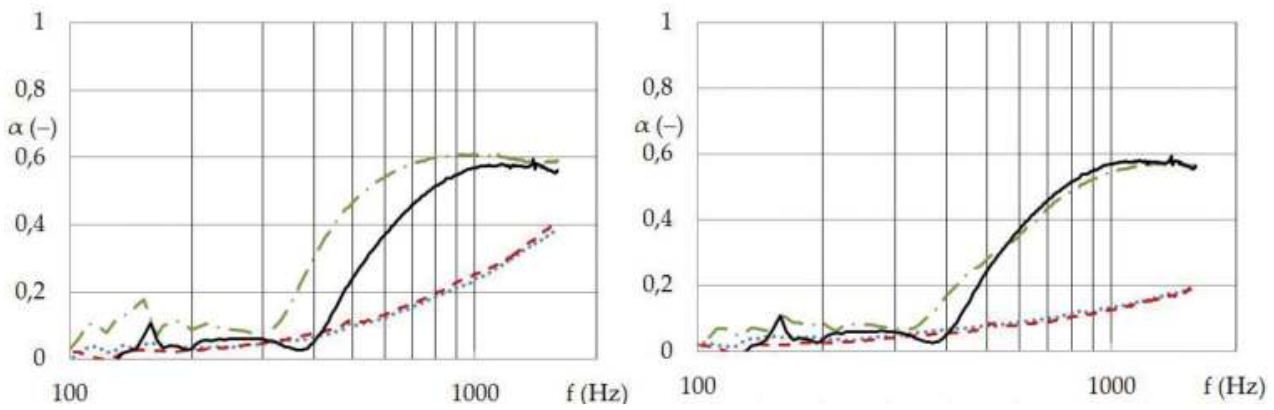

Figure 9. Frequency dependence of the sound absorption coefficient; quad perforated plate (9/11) covered by the nanofibrous membrane of $0.2 \mathrm{~g} / \mathrm{m}^{2}$ on a carrier of $25 \mathrm{~g} / \mathrm{m}^{2}$. The air gap between the 1-mm thick panel and the wall is $20 \mathrm{~mm}$ (black): the same nanofibers-covering perforated plate filled (green - dash-dotted) by the foam (left) or fleece (right); the same separated plate filled (red - dashed) by the foam (left) or fleece (right); the separated fillings (blue dotted) of foam (left) or fleece (right). The thickness of the whole acoustic system is $20 \mathrm{~mm}$ in all configurations.

membrane resonators covering the mass of Helmholtz's resonator obstruct the sound absorption inside the cavity.

The individual perforated plates in a form of cavity resonators should be filled for sound absorption of higher frequencies. Figure 9 shows the comparison of acoustic system consisting of nanofibers-covering quad perforated plate and the same perforated plate without covering but filled. The filling has been chosen from the standard sound absorbers line. The first is melamine foam of $9.5 \pm 1.5 \mathrm{~kg} / \mathrm{m}^{3}$ and 20-mm thickness (Figure 9 on the left) and the second polyester fleece of $24 \mathrm{~kg} / \mathrm{m}^{3} \pm 10 \%$ and 20-mm thickness (Figure 9 on the right). From the comparison, it can be seen that the inferior sound absorption results if the perforated plate is filled (red - dashed curve) in comparison with nanofibers covering the same perforated plate without filling (black curve). Then, the resonance capability of nanofibrous membrane more than compensates a mass of filling. Regarding the applicability of nanofibers-covering perforated plate, the gap between the panel and the wall can be used for light or audio installation. If the nanofiber-covering plate is filled (green - dash-dotted curve), then the sound absorption is slightly better than that of non-filled. However, the benefit of air gap outweighs the nominal sound absorption growth.

\section{Conclusions}

The resonance ability of nanofibrous layer has been verified in the last author's paper. The membrane has been exposed to plane sinusoidal sound wave and its deflection was picked by the high-speed digital camera. The resonant peaks of oscillating nanofibrous membrane as well as homogeneous membrane occur around 70-100, 300-400, and 550-600 Hz depending on their parameters. The calculated resonant frequency of the perforated plate is around $2-2.5 \mathrm{kHz}$. The sound absorption peaks of nanofibers-covering perforated plate are around $500 \mathrm{~Hz}$. From the comparison of resonant frequencies perforated plate in a form of Helmoltz's resonator, 
separate nanofibrous layer in a form of membrane resonator, and final nanofibers-covering perforated plate, the major effect of the resonant frequency of the nanofibrous layer together with a distance of the final plate from the wall can be seen.

The diameter of nanofibers, the basis weight, and the polymer of the nanofibrous membrane as well as the shape, size, and span of perforations of Helmholtz's resonator affect the sound absorption behavior of acoustic element.

The two applied nanofibrous membranes have not almost any effect on sound absorption. The improvement would be redundant.

\section{Acknowledgements}

The results of this project LO1201 were obtained through the financial support of the Ministry of Education, Youth and Sports in the framework of the targeted support of the "National Programme for Sustainability I" and the OPR\&DI project Centre for Nanomaterials, Advanced Technologies and Innovation CZ.1.05/2.1.00/01.0005. Particularly the work was supported by the Technology Agency of the Czech Republic within the project "Broadband components with resonant nanofibrous membrane for room acoustics" no. TH02020524. I also thank my doctoral student Tomas Ulrich for measurement support.

\section{Author details}

Klara Kalinova

Address all correspondence to: klara.kalinova@tul.cz

Technical University of Liberec, Czech Republic

\section{References}

[1] Kolmer F, Kyncl J. Prostorová Akustika (in Czech). Prague: SNTL Praha; 1980. p. 244. ISBN: 04-514-80

[2] Merhaut J. Teoretické Základy Elektroakustiky (in Czech). Vol. 3. Prague: Academia; 1976. p. 325

[3] Škvor Z. Akustika a Elektroakustika (in Czech). Vol. 1. Prague: Academia; 2001. p. 527. ISBN: 80-200-0461-0

[4] Asmatulu R, Khan W, Yildirim MB. Acoustical Properties of Electrospun Nanofibers for Aircraft Interior Noise Reduction [Online]. B.m.: ASME. 2009;2009:223-227. ISBN: 978-07918-4388-8. DOI: 10.1115/IMECE2009-12339 
[5] Kalinová K. Nanofibrous resonant membrane for acoustic applications. Journal of Nanomaterials. 2011;2011:1687-4129. ISSN: 1687-4110. DOI: 10.1155/2011/265720

[6] Kimihiro S, Kiyama M, Morimoto M, Takahashi D. Sound absorption of a cavity-backed membrane: A step towards design method for membrane-type absorbers. Applied Acoustics. 1996;49(3):237-247. ISSN: 0003682X-4. DOI: 10.1016/S0003-682X(96)00025-4

[7] Kimihiro S, Morimoto M, Yairi M. A note on the relationship between the sound absorption by microperforated panels and panel/membrane-type absorbers. Applied Acoustics. 2009;70(8):1131-1136. ISSN 0003-682X. DOI: http://dx.doi.org/10.1016/j.apacoust.2009.03. 003

[8] Hoda SS. Factors influencing acoustic performance of sound absorptive materials. Australian Journal of Basic and Applied Sciences. 2009;3(4):4610-4617. ISSN 1991-8178

[9] Rabbi A, Nasouri K, Shoushtari AM, Haji A. Fabrication of electrospun polyacrylonitrile and polyurethane nanofibers for sound absorption. In: 6th TEXTEH International Conference; October 17-18, 2013; Bucharest, Romania. 2013. pp. 233-241. DOI: https://www. academia.edu/download/45476648/FABRICATION_OF_ELECTROSPUN_POLYACRYLON IT20160509-22247-3x9zsf.pdf

[10] Randeberg RT. Perforated panel absorbers with viscous energy dissipation enhanced by orifice design [PhD thesis, dissertation]. NTNU Trondheim, 2000. Available from: https:// brage.bibsys.no/xmlui/bitstream/handle/11250/249798/125365_FULLTEXT01.pdf?sequence= 1\&isAllowed $=\mathrm{y}$

[11] Kobayashi Y, Hase S. Examination of a resonator system for sound power absorption by hall chairs in the low-frequency range. The Journal of the Acoustical Society of America. 1996;99:2459

[12] Stauskis VJ. Sound absorption by a large-size isolated acoustic resonator with a cylindrical cavity. The Journal of the Acoustical Society of America. 1998;103(5):306

[13] Chanaud RC. Effects of geometry on the resonance frequency of Helmholtz resonators. Journal of Sound and Vibration. 1994;178(3):337-348. DOI: 10.1006/jsvi.1994.1490

[14] Kourosh T, Per G. Measurement of milling tool vibrations during cutting using laser vibrometry. International Journal of Machine Tools and Manufacture. 2008;48(3-4):380387. ISSN: 08906955. DOI: 10.1016/j.ijmachtools.2007.09.009

[15] Morteza K, Lu W, Dobrev I, Cheng JT, Furlong C, Rosowski JJ. Digital holographic measurements of shape and three-dimensional sound-induced displacements of tympanic membrane. Optical Engineering. 2013;52(10):101916. ISSN: 0091-3286. DOI: 10.1117/1.OE.52.10.101916

[16] Elisabeth IC, Döllinger M, Schuster M, Eysholdt U, Bohr C. Multiparametric analysis of vocal fold vibrations in healthy and disordered voices in high-speed imaging. Journal of Voice. 2011;25(5):576-590. ISSN: 08921997. DOI: 10.1016/j.jvoice.2010.04.004 
[17] Weizhuo W, Mottershead JE, Ihle A, Siebert T, Schubach HR. Finite element model updating from full-field vibration measurement using digital image correlation. Journal of Sound and Vibration. 2011;330(8):1599-1620. ISSN: 0022460X. DOI: 10.1016/j.jsv.2010. 10.036

[18] Ishizu K, Yasui T, Ohashi M, Matsumoto N, Komune S. High-speed video analysis of acoustically oscillated guinea pig stapes. The Journal of Laryngology \& Otology. 2015;129(2):S33-S37. ISSN: 0022-2151. DOI: 10.1017/S0022215114002357

[19] Qican Z, Su X. High-speed optical measurement for the drumhead vibration. Optics Express. 2005;13(8):3110. ISSN: 1094-4087. DOI: 10.1364/OPEX.13.003110

[20] Siebert T, Wood R, Splitthof K. High speed image correlation for vibration analysis. Journal of Physics: Conference Series. 2009;181:12064. ISSN: 1742-6596. DOI: 10.1088/ 1742-6596/181/1/012064

[21] David Asael G-H, Perez Lopez C, Mendoza Santoyo F, Tellez-Quinones A, Aguayo DD. Optical characterization of a semisolid membrane by high speed interferometry. Optoelectronics and Advanced Materials, Rapid Communications. 2014;8(5-6):382-386. ISSN: 1842-6573

[22] Majid N, Siddiqui K, Dargahi J. Measurement of the acoustic velocity field of nonlinear standing waves using the synchronized PIV technique. Experimental Thermal and Fluid Science. 2008;33(1):123-131. ISSN: 08941777. DOI: 10.1016/j.expthermflusci.2008.07.013

[23] Ozturk MK, Kalinova K, Nergis B, Candan C. Comparison of resonant frequency of nanofibrous membrane and homogenous membrane. Textile Research Journal. 2013;83(20): 2204-2210. DOI: $10.1177 / 0040517513490064$

[24] Klara K, Ozturk MK, Komarek M. Open and closed tube method for determination of resonance frequencies of nanofibrous membrane. The Journal of the Textile Institute. 2016;107(8):1068-1078. DOI: 10.1080/00405000.2015.1083353

[25] Maly M, Sejak P, et al. Method for Application of Liquid Polymeric Material onto Spinning Cords and a Device for Production of Nanofibers Through Electrostatic Spinning. Patent 2012, WO2012139533; 2012 
